\title{
Clinical Applications for Tissue Engineering in Rhinology
}

\author{
Emmanuel Prokopakis ${ }^{1}\left(\mathbb{D}\right.$, Maria Doulaptsi $^{1}$ (D), Alexander Karatzanis ${ }^{1}$ (D), Hideyuki Kawauchi ${ }^{2}$ (D) \\ Review ${ }^{1}$ Department of Otorhinolaryngology - Head and Neck Surgery, University of Crete School of Medicine, Crete, Greece \\ ${ }^{2}$ Department of Otorhinolaryngology, University of Shimane School of Medicine, Shimane, Japan
}

\begin{abstract}
Tissue engineering implies a number of established techniques in several fields in medicine. A thorough review of current clinical applications for tissue engineering in rhinology is addressed. Current status, as well as, published in vivo studies is presented. Moreover, relevant clinical applications and future perspectives of tissue engineering are demonstrated. There is
\end{abstract}

ORCID IDs of the authors: E.P. 0000-0002-1208-1990; M.D. 0000-0001-9011-0315;

A.K. 0000-0003-3196-2176; H.K. 0000-0003-4161-2591.

Cite this article as: Prokopakis E, Doulaptsi $M$, Karatzanis A, Kawauchi H. Clinical Applications for Tissue Engineering in Rhinology. Turk Arch Otorhinolaryngol 2019; 57(1):39-41.

Corresponding Author: Emmanuel Prokopakis; eprokopakis@gmail.com Received Date: 21.10.2018 Accepted Date: 06.03.2019 (c) Copyright 2019 by Official Journal of the Turkish Society of Otorhinolaryngology and Head and Neck Surgery Available online at www.turkarchotolaryngol.net DOI: 10.5152/tao.2019.3889

\section{Introduction}

Tissue engineering employs a biocompatible scaffold, with or without cells, to restore or establish normal function in tissues (1). In addition to cells, the scaffolds can be supplemented with various growth factors. A scaffold employed in tissue engineering should ideally be biocompatible, biodegradable, possess appropriate mechanical structure, and be clinically and commercially available (2).

Tissue engineering may be applied to a number of operations in rhinology, including augmentative and reconstructive rhinoplasty, nasal septal repair, alar nasal reconstruction, nasal deformities and craniofacial reconstruction. When developing tissue-engineering techniques for such procedures, the main focus should be given on cartilage. Hyaline cartilage, such as that found in the nose, microscopically consists of chondrocytes. Derived from mesenchymal stem cells (MSCs), chondrocytes are responsible for producing the extracellular matrix that mainly consists of Type II collagen and proteoglycans. The avascular, aneural and alymphatic nature of hyaline cartilage result in it having a limited capacity for intrinsic healing and regeneration $(3,4)$. Therefore, the use of autolo- a lack of high quality clinical studies in the literature regarding the role of tissue engineering in the rhinology field. Further research is needed to translate this concept from bench to bedside.

Keywords: Cartilage, in vivo, reconstructive surgery, rhinoplasty gous transplants or synthetic materials is required to replace the tissue.

Currently, the gold standard for the reconstruction of cartilage defects in many surgical operations such as otorhinolaryngology and orthopaedics is autologous cartilage, however, donor site morbidity, limited availability, and the need for complex surgical operations have diminished its use (3). Cartilage tissue engineering has emerged as a promising method to replace damaged or missing tissue (5).

\section{Clinical and Research Consequences}

\section{In vivo studies}

In vivo studies in animals play a vital role in research and provide the foundations for clinical trials. The ideal material for nasal septum reconstruction is yet to be identified. Bermueller et al. (6) investigated the use of marine collagen scaffolds for nasal cartilage repair. The rationale for employing marine collagen is due to the fact that most marine species are invertebrates and therefore will not possess substances that are harmful to humans such as transmissible diseases. The marine collagen 
scaffolds were seeded with nasal chondrocytes from rats and humans and implanted in an orthotopic rat model. In vitro results demonstrated no cytotoxic reactions. Results from the in vivo study showed that the scaffolds displayed appropriate properties for regeneration of nasal septal cartilage and were able to prevent septal perforation (6).

Another study by the same research group investigated the use of a decellularised collagen matrix. The scaffolds were evaluated in vitro by seeding with rat nasal septum chondrocytes and did not show any cytotoxic properties. Moreover, implantation into a rat model showed that decellularised xenogeneic collagen scaffolds were able to integrate well into septal cartilage defects (7). Both scaffolds investigated by this research group showed promising in vitro and in vivo characteristics. They were both able to prevent the development of septal perforations and therefore may provide a solution for nasal septum reconstruction.

Another field of rhinology where tissue engineering may be employed is in augmentative and reconstructive rhinoplasties. Whilst autologous grafts are commonly used in rhinoplasty operations, donor site morbidity limits its use. Furthermore, the use of synthetic materials may result in implant infection or allocation as well as an unnatural appearance (8). Preliminary use of scaffolds in this field showed great promise. These consisted of a poly (lactic-co-glycolic acid) base with alginate containing gelatin microspheres, and enriched with human transforming growth factor beta- 3 in varying doses. They were implanted onto the native nasal cartilage in a rat model of augmentation rhinoplasty and led to the formation of cartilage-like tissue (8).

\section{Clinical applications}

Lately, tissue-engineering therapeutic approaches in rhinology have been employed in human trials with promising results. Non-melanoma skin cancers are the most common malignant skin neoplasm in Caucasians. These lesions arise most frequently in the nasal alar lobule (9). Surgery is the treatment of choice, however, due to its thick and sebaceous skin and reduced mobility, reconstruction of the nasal ala poses a surgical challenge (10).

The standard material for reconstructing the nasal alar lobule following two-layer excision of skin cancer is autologous cartilage harvested from the nasal septum, ear or rib; however, donor site morbidity limits its use (11). The use of engineered autologous cartilage grafts was tested in five cases in a Swiss study. It involved harvesting autologous chondrocytes from the subjects' nasal septum and expanding and seeding them onto Collagen Type 1 and 3 scaffolds prior to implantation. A one-year follow up showed that the reconstructed tissues displayed similar properties to native alar lobule and patients reported satisfaction with aesthetic and functional outcomes (11).

Gentile et al. (12) investigated the use of a combination of autologous chondrocyte micrografts and platelet-rich plasma (PRP) in eleven patients with external nasal valve collapse. PRP is extracted from the patient's blood and provides a source of growth factors. Moreover, this combined material could be sub- cutaneously injected therefore offering a minimally invasive approach (12). The analysis of excised fragments from two patients revealed that healthy cartilage similar to normal tissue formed (12). Longer follow up studies and larger sample sizes are still required for stronger conclusions.

\section{Future perspectives}

Cartilage tissue engineering classically uses scaffolds with or without cells; however the concept of scaffold-free cartilage tissue engineering is emerging. The rationale for scaffold free tissue engineering is because the use of exogenous materials can inflict host immunogenic responses, which can lead to implant failure (13). This process involves culturing human nasoseptal chondrocytes in a bioreactor and varying the medium composition and amounts of growth factors (14). Studies have shown that this technique is able to generate cartilage similar to that of native human cartilage. However, only in vitro studies are available (13, 14). To date, there is no information in the literature about the performance of these constructions in animal models.

One of the limitations of current tissue engineered constructions is a lack of precision in their shape. This may be overcome with the use of three-dimensional (3D) printing also known as additive manufacturing. This technique is able to create complex patient-specific constructions using biocompatible scaffolds and cells (15). Poly (glycolic acid)/poly-L-lactic acid (PGA/PLLA) is a common biocompatible biomaterial that can be employed to construct scaffolds with the aid of 3D printing. Xu et al. (16) harvested human nasal cartilage and seeded them onto PGA/ PLLA scaffolds. These were cultured in vitro before implanted into subcutaneous tissue of nude mice. It was found that 3D printed, tissue engineered constructions could produce structures with similar histological and biomechanical properties with the human nasal alar cartilage. To date, the use of 3D printed tissue engineering approaches is a promising solution that needs to be reported in clinical studies. It is noticeable, that $3 \mathrm{D}$ printing techniques have been recently applied to facilitate preoperative and postoperative assessment in rhinoplasty (17).

In addition to chondrocytes, stem cells have been considered as another source of cells for cartilage tissue engineering. The sources of stem cells include tissue-derived stem/progenitor cells, human embryonic stem cells and more recently human induced pluripotent stem cells. These sources stem cells for application in rhinology has been reviewed by Vats and Birchall (18). However, stem cells have their limitations. The process of chondrocyte formation from stem cells is not yet fully understood. Additionally, there is a risk of teratoma formation when implanted in vivo (19).

\section{Conclusion}

There are a number of in vitro studies published in the literature that aim to apply tissue engineering techniques to rhinologic surgical procedures. However, only limited in vivo studies and clinical trials have been published highlighting the difficulty to translate these concepts from bench to bedside. Crosstalk among research groups around the world investigating this multidisci- 
plinary field would be appropriate in order to discuss the most promising in vitro and in vivo studies and decide what results may be applied into human trials.

Peer-review: Externally peer-reviewed.

Author Contributions: Concept - E.P., H.K.; Design - M.D., A.K., E.P.; Supervision - E.P., H.K., A.K.; Resource - M.D., A.K., H.K.; Materials - M.D., A.K.; Data Collection and/or Processing - E.P., M.D., A.K.; Analysis and/or Interpretation - E.P., H.K., A.K.; Literature Search - M.D., E.P., H.K.; Writing - M.D., E.P.; Critical Reviews - E.P., M.D., A.K., H.K.

Conflict of Interest: The authors have no conflicts of interest to declare.

Financial Disclosure: The authors declared that this study has received no financial support.

\section{References}

1. Wormald JCR, Fishman JM, Juniat S, Tolley N, Birchall MA. Regenerative medicine in otorhinolaryngology. J Laryngol Otol 2015; 129: 732-9. [CrossRef]

2. O'Brien FJ. Biomaterials \& scaffolds for tissue engineering. Mater Today 2011; 14: 88-95. [CrossRef]

3. Chung $\mathrm{C}$, Burdick JA. Engineering cartilage tissue. Adv Drug Deliv Rev 2008; 60: 243-62. [CrossRef]

4. Dinis PB, Dinis M, Gomes A. Psychosocial consequences of nasal aesthetic and functional surgery: a controlled prospective study in an ENT setting. Rhinology 1998; 36: 32-6.

5. Kaliva M, Chatzinikolaidou M, Vamvakaki M. Applications of Smart Multifunctional Tissue Engineering Scaffolds. Wang Q editor. Smart Materials for Tissue Engineering: Applications. The Royal Society of Chemistry; 2017.p.1-38. [CrossRef]

6. Bermueller C, Schwarz S, Elsaesser AF, Sewing J, Baur N, von Bomhard A, et al. Marine collagen scaffolds for nasal cartilage repair: prevention of nasal septal perforations in a new orthotopic rat model using tissue engineering techniques. Tissue Eng Part A 2013; 19: 2201-14. [CrossRef]

7. Elsaesser AF, Bermueller C, Schwarz S, Koerber L, Breiter R, Rotter N. In vitro cytotoxicity and in vivo effects of a decellularized xenogeneic collagen scaffold in nasal cartilage repair. Tissue Eng Part A 2014; 20: 1668-78. [CrossRef]

8. Mendelson A, Ahn JM, Paluch K, Embree MC, Mao JJ. Engineered nasal cartilage by cell homing: a model for augmentative and reconstructive rhinoplasty. Plast Reconstr Surg 2014; 133: 1344-53. [CrossRef]

9. Wettstein R, Erba P, Farhadi J, Kalbermatten DF, Arnold A, Haug $\mathrm{M}$, et al. Incomplete excision of basal cell carcinoma in the subunits of the nose. Scand J Plast Reconstr Surg Hand Surg 2008; 42: 92-5. [CrossRef]

10. Ortins-Pina A, Teixeira AI, Sanches M, Gouveia AI, Filipe PL, Silva JM. Nasal ala reconstruction: Surgical conundrum. J Cutan Aesthet Surg 2017; 10: 55-8. [CrossRef]

11. Fulco I, Miot S, Haug MD, Barbero A, Wixmerten A, Feliciano S, et al. Engineered autologous cartilage tissue for nasal reconstruction after tumour resection: an observational first-in-human trial. The Lancet 2014; 384(9940):337-46. [CrossRef]

12. Gentile P, Scioli MG, Bielli A, Orlandi A, Cervelli V. Reconstruction of alar nasal cartilage defects using a tissue engineering technique based on a combined use of autologous chondrocyte micrografts and platelet-rich plasma. Plast Reconstr Surg - Glob Open 2016; 4(10): e1027. [CrossRef]

13. Akbari P, Waldman SD, Propst EJ, Cushing SL, Weber JF, Yeger $\mathrm{H}$, et al. Generating mechanically stable, pediatric, and scaffold-free nasal cartilage constructs in vitro. Tissue Eng Part C Methods 2016; 22: 1077-84. [CrossRef]

14. Chiu LL, To WT, Lee JM, Waldman SD. Scaffold-free cartilage tissue engineering with a small population of human nasoseptal chondrocytes. Laryngoscope 2017; 127:E91-9. [CrossRef]

15. Rehmani SS, Bhora FY. Current state of 3D printing in tissue engineering. J 3D Print Med 2017; 1: 77-9. [CrossRef]

16. Xu Y, Fan F, Kang N, Wang S, You J, Wang H, et al. Tissue engineering of human nasal alar cartilage precisely by using three-dimensional printing. Plast Reconstr Surg 2015; 135: 451-8. [CrossRef]

17. Lekakis G, Claes P, Hamilton GS, Hellings PW. Three-dimensional surface imaging and the continuous evolution of preoperative and postoperative assessment in rhinoplasty. Facial Plast Surg 2016; 32: 88-94. [CrossRef]

18. Vats A, Birchall M. Stem cells and regenerative medicine: potentials and realities for rhinology. Rhinology 2010; 48: 259-64. [CrossRef]

19. Oseni AO, Butler PE, Seifalian AM. Nasal reconstruction using tissue engineered constructs. Ann Plast Surg 2013; 71: 238-44. [CrossRef] 